\title{
Coupled Anharmonic Vibrational Dynamics of the Hydrogen Bond in Binary Complexes
}

\author{
Manuel Goubet, Bruno Madebène, and Marius Lewerenz ${ }^{*}$
}

\begin{abstract}
We report anharmonic vibrational analyses of the coupling between the red-shifted high frequency HFstretching vibrations and low frequency intermolecular modes in the hydrogen-bonded complexes thiirane-HF and $\mathrm{H}_{2} \mathrm{O}-\mathrm{HF}$. The important non-diagonal anharmonicities found in high-level ab initio calculations are consistent with recent experimental results. The physical origin of the coupling mechanism implies that a physically correct treatment of hydrogen-bond induced vibrational red shifts requires a vibrational treatment far beyond the customary one-dimensional approach combined with large basis set electronic structure calculations.
\end{abstract}

Keywords: Hydrogen-bonded complexes $\cdot$ Hydrogen fluoride $\cdot$ Thirane $\cdot$ Vibrational dynamics $\cdot$ Water

\section{Introduction}

The importance of hydrogen bonding for the structure of liquids and the threedimensional structure of biopolymers has been known for a very long time and has motivated an enormous number of experimental and theoretical studies [1]. The most detailed information on the vibrational spectroscopy and its relation to the shape of the potential energy is available for small complexes like $(\mathrm{HF})_{2}[2],\left(\mathrm{H}_{2} \mathrm{O}\right)_{2}$ [3], and other complexes involving small molecules [4]. The combination of advanced electronic structure methods [5] and more or less complete coupled anharmonic vibrational treatments [6] of these systems has reached a high degree of sophistication and is in very close agreement with the best available experimental results [7][8]. The dynamical analysis of these systems has revealed important effects of the excitation of intramolecular modes on the intermolecular interaction. The commonly observed and physically motivated strengthening of the

${ }^{*}$ Correspondence: Prof. M. Lewerenz LADIR, UMR 7075, Bât F74, Bte 49,

Université Pierre et Marie Curie

F-75252 Paris Cedex 05, France

Tel.: +33 144273023

Fax: +33144273021

E-Mail: lewerenz@spmol.jussieu.fr interaction and the resulting tightening of the complexes leads among other effects to the strong vibrational dependence of the tunneling splitting in $(\mathrm{HF})_{2}$. Qualitatively this coupling is easy to understand since at least the electrostatic component of the intermolecular interaction [9] increases upon excitation of the HF donor due to its enhanced dipole moment in the vibrationally excited state.

A characteristic feature in the vibrational spectra of hydrogen-bonded complexes is a strong shift of the donor stretching frequency towards lower values usually accompanied by a strong enhancement of the vibrational cross section and by broadening due to predissociation [10]. A lot of effort has gone into the analysis of this vibrational red shift and its relation to the strength of the hydrogen bond. The importance of coupling between high- and lowfrequency modes in these systems has long been recognized [11] and is a key element in the final state distribution following vibrational predissociation [12]. It is therefore surprising that with very few exceptions [13][14] the calculation of vibrational red shifts in hydrogen bonded complexes containing more than a few atoms has remained limited to the harmonic level even though the global shape of this interaction has attracted some interest [15]. While this approach can be justified by a relative success at predicting the change of the vibrational frequency, the absolute frequencies are far too high and of course cannot reflect the increased anharmonicity of the donor vibrations following complexation, which is a well-known effect [1]. This is a clear case of significant compensation of major errors in the electronic structure treatment and the modeling of the vibrational dynamics. It is dangerous to rely on systematic error cancellation since these two errors have no clear physical connection and a better understanding of hydrogen bonding can be only reached by clear identification of the deficits of current electronic structure methods and parallel efforts towards more complete vibrational calculations. Inclusion of only diagonal anharmonic effects [16] is insufficient due to the experimentally evident and physically motivated coupling and again succeeds at reproducing red shifts only by the use of the improper MP3 method which very seriously overestimates the anharmonic frequencies of the HF monomer. Focusing theoretical efforts exclusively on the prediction of the donor shift neglects the important structural consequences of the complete topography of this interaction.

Our group has embarked on a combined experimental and theoretical program which aims at the observation of intermolecular modes and their coupling to the donor stretch through hot band progressions in the gas phase and direct studies in cryogenic matrices [17] and coupled anharmonic vibrational studies of the relevant potential surfaces. The present article reports preliminary theoretical results on two complexes which are representative of the small dimer limit with pronounced tunnel- 
ing $\left(\mathrm{HF}-\mathrm{H}_{2} \mathrm{O}\right)$ and the large molecule case with essentially quenched tunneling (thiirane-HF) in spite of the existence of two equivalent arrangements.

The complex thiirane-HF is a representative of medium-strength hydrogen-bonded complexes involving organic ethers or thioethers. Thirane, $\left(\mathrm{CH}_{2}\right)_{2} \mathrm{~S}$, is a threemembered ring compound which in extended contact with HF undergoes a ring-opening reaction. This class of systems presents several theoretical and experimental challenges due to its size and number of degrees of freedom [17]. The dynamics of these complexes are, however, definitely closer to hydrogen bonding in biomolecules than the paradigmatic small complexes $(\mathrm{HF})_{2}$, $\left(\mathrm{H}_{2} \mathrm{O}\right)_{2}$ etc. and deserve attention in order to understand the full shape of the local intermolecular potential. Complexes with aliphatic compounds are not easily accessible for high-resolution laser techniques routinely applied for hydrogen-bonded complexes with aromatic constituents [18] due to the absence of a good vis/UV-chromophore. Even though these complexes, which often exist in thermodynamic equilibrium with the monomers, have been investigated with infrared spectroscopic techniques for a long time [19] high-resolution studies and unambiguous assignments of the component bands have become possible only due to advances in molecular jet techniques [17][20]. The detailed analysis of the latter experiments has unveiled a global coupling scenario involving the red-shifted donor stretch and all low-frequency intermolecular modes corresponding to frustrated translations. The insufficient thermal occupation of excited states of the remaining higher frequency intermolecular modes (around $700 \mathrm{~cm}^{-1}$ ) arising from frustrated rotations of the HF unit has so far prevented the observation of coupling via hot bands accompanying the HF stretch band.

We are interested in $\mathrm{HF}-\mathrm{H}_{2} \mathrm{O}$ as the first member of the series of complexes HF$\left(\mathrm{H}_{2} \mathrm{O}\right)_{n}$ which is expected to exhibit sizedependent competition between formation of a hydrogen bond and acid dissociation [13][21]. This subject has recently attracted a lot of attention motivated by the importance of acid dissociation on nanocrystals in atmospheric science. Besides some earlier theoretical studies [24] the $\mathrm{HF}-\mathrm{H}_{2} \mathrm{O}$ complex is known from matrix isolation [25], microwave [26] and early infrared studies [27] since it exists in thermodynamic equilibrium with the monomers in the gas phase. We are currently undertaking efforts to observe the vibrational spectrum of HF$\mathrm{H}_{2} \mathrm{O}$ in a free jet experiment. A good entry point into the earlier literature on water heterodimers can be found in [23] and [28]. Several complementary studies [29] are available for the $\mathrm{HF}-\mathrm{H}_{2} \mathrm{~S}$ complex which is in many ways intermediate between the two complexes discussed here. The vibrational dynamics of $\mathrm{HF}-\mathrm{H}_{2} \mathrm{O}$ is expected to exhibit the same coupling phenomena as observed in thiirane-HF and other systems but with the additional twist of a very pronounced tunneling splitting which will sensitively depend on the excitation of the HF donor.

\section{Theoretical Methods}

\subsection{Electronic Structure Calcula- tions}

The electronic structure calculations for thiirane-HF were carried out with the Gaussian-98 program package [30]. The proper description of intermolecular interactions requires basis sets including diffuse functions. Due to the important size of this complex the basis set was limited to the aug-cc-pVTZ type proposed by Dunning and coworkers [31] which provides a good compromise between computational effort and tolerable basis set superposition errors (BSSE). It is interesting to note that for this type of augmented basis set the standard counterpoise correction appears to overestimate the actual size of the BSSE such that corrected dissociation energies for hydrogen-bonded dimers are further away from the estimated basis set limit than uncorrected ones [31]. While exploratory calculations were performed with several other methods, all data reported here are based on MP2 calculations. The counterpoise correction was applied only to the dissociation energy but not to the entire surface. We did, however, test the sensitivity of the vibrational results to this correction and found effects on vibrational energies never exceeding $5 \mathrm{~cm}^{-1}$ and smaller for the transition frequencies. The effect is obviously largest for the intermolecular stretch.

The calculations for the binary complex $\mathrm{HF}-\mathrm{H}_{2} \mathrm{O}$ were performed with the MOLPRO suite of programs [32] using the augcc-pVTZ basis set and MP2, CCSD(T) and MR-CI methods. The use of larger basis sets would be affordable for $\mathrm{HF}-\mathrm{H}_{2} \mathrm{O}$ but not for larger complexes $\mathrm{HF}-\left(\mathrm{H}_{2} \mathrm{O}\right)_{n}$ which we are studying in the context of acid dissociation in clusters. A more detailed account of our results for several isotopomers and various tests of basis sets and methods will be published elsewhere [33][34].

We paid particular attention to the reproduction of known properties of the monomers as a minimum requirement for an acceptable description of the complexes. The performance of the chosen combination of basis set and computational method was tested by the calculation of anharmonic vibrational energies and expectation values for other properties which can be directly compared with experiment instead of the more common procedure to compare electronic structure results with values resulting from extrapolations of experimental data. Besides a good reproduction of the anharmonic HF frequency and the expectation value of the rotational constants the importance of electric properties for intermolecular interactions led us to demand satisfactory results for the dipole moment curve and the resulting dipole moment expectation values and transition moments as an important selection criterion. The availability of dipole moment surfaces allows the investigation of the respective importance of the increased anharmonicity and the modified dipole surface for the observed enhancement of vibrational cross sections upon complexation. Our results indicate that the observed enhancement results in part from an increased slope of the dipole moment curve, but more importantly from a pronounced curvature of the latter which in combination with the strong anharmonicity is responsible for almost half the effect.

\subsection{Choice of Coordinates}

A complete and numerically exact vibrational treatment of $\mathrm{HF}-\mathrm{H}_{2} \mathrm{O}$ for a given potential surface is currently not possible and will probably remain impossible for thiirane-HF for a very long time. We have therefore decided in a first step to investigate subspaces of coupled coordinates. The equilibrium geometry of both complexes is similar to a plane defined by $\mathrm{H}_{2} \mathrm{O}$ and thiirane, respectively, and the $\mathrm{HF}$ unit in the symmetry plane of the acceptor forming an almost linear hydrogen bond (Fig. 1). There are clearly two equivalent arrangements separated by a barrier at the planar arrange-

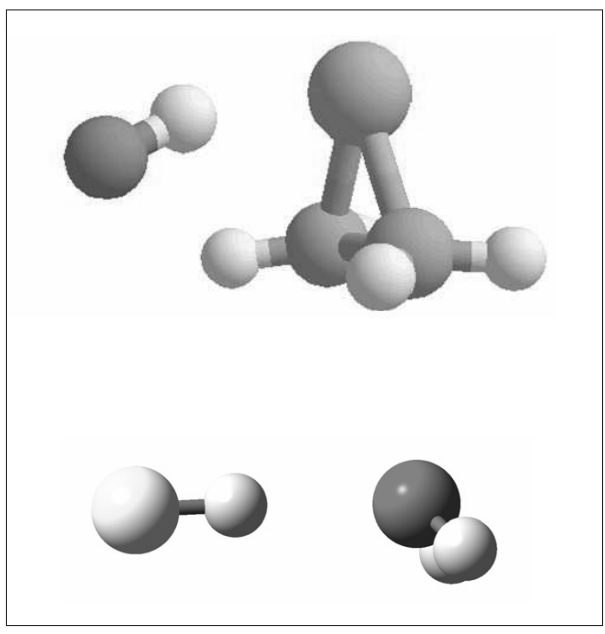

Fig. 1. Equilibrium arrangement of the complexes thirane- $\mathrm{HF}$ (top) and $\mathrm{HF}-\mathrm{H}_{2} \mathrm{O}$ (bottom).

\section{ment.}

The distant possibility of a full-dimensional treatment of $\mathrm{HF}-\mathrm{H}_{2} \mathrm{O}$ and the low barrier (electronic barrier to planarity 157 $\mathrm{cm}^{-1}$ ) leading to pronounced tunneling has motivated us to scan the potential surface 
along generalized Jacobi coordinates such that the intermolecular distance $R$ corresponds to the distance between the centers of mass of $\mathrm{HF}$ and $\mathrm{H}_{2} \mathrm{O}$. This coordinate system belongs to a class which leads to particularly compact and elegant kinetic energy expressions [35]. The explorations presented here concern the HF interatomic distance $r, R$ as defined above, and four intermolecular angles describing the hindered rotation of HF (librational modes) and the intermolecular bending and tunneling motion.

For thiirane-HF and -DF we chose to explore the potential surface along normal coordinates which take into account quadratic coupling with ring modes which are not dealt with explicitly. The large barrier to planarity of almost $1100 \mathrm{~cm}^{-1}$ leads to moderate vibrational amplitudes which make normal coordinates applicable. The surface was scanned along the relevant normal coordinates by displacing all atoms according to the transformation matrix between cartesian and normal coordinates resulting from the digitalization of the mass weighted $a b$ initio cartesian force constant matrix. The resulting normal coordinates for the motions of the thiirane-HF complex are very similar to the internal coordinates chosen for $\mathrm{HF}-\mathrm{H}_{2} \mathrm{O}$ due to strongly local vibrational dynamics which provides further justification for subspace treatments.

\subsection{Vibrational Analysis}

Even though anharmonic effects in weakly bound complexes can be very large it is not unreasonable to try to represent the dependence of the vibrational energy on a set of quantum numbers $\underline{\mathrm{v}}=\mathrm{v}_{1}, \mathrm{v}_{2}, \ldots$ at least for the modest excitations of interest in the present context by the conventional

termolecular modes $q$ upon excitation of the donor stretch mode $v_{\mathrm{s}}$ implies that the corresponding off-diagonal anharmonicities $x_{s q}$ are negative. Another way of looking at the same phenomenon is the increase of the donor stretch frequency upon excitation of the intermolecular modes. This is easy to rationalize for the strongly anharmonic intermolecular stretch whose excitation significantly increases the expectation value of the intermolecular distance and thereby brings the donor closer to its asymptotic

free vibration. This potential coupling is accompanied by a smaller dynamic effect arising from the change of the moment of inertia of HF upon excitation which affects mainly the intermolecular libration (frustrated rotation) modes.

Eqn. (2) clearly shows that in the present situation neglect of coupling terms will underestimate the donor stretch frequency $v_{\mathrm{s}}$ by an amount $1 / 2 \sum_{j \neq s} x_{s j}$. The analysis of several experimental data sets [8][17] suggests that the $x_{s j}$ are typically about $-10 \%$ of the frequency $v_{j}$. With typical intermolecular frequencies in the range $100 \mathrm{~cm}^{-1}$ for bending modes $\delta, 250 \mathrm{~cm}^{-1}$ for intermolecular stretching $\sigma$, and $650 \mathrm{~cm}^{-1}$ for libration modes $l$ this provides a correction around $90 \mathrm{~cm}^{-1}$. In other words, a one-dimensional anharmonic treatment will systematically overestimate the hydrogen-bond redshift by about $90 \mathrm{~cm}^{-1}$, which is roughly $20 \%$ of the total shift for medium-strength hydrogen bonds.

Since the analysis of experimental data provides values for the various $x_{i j}$ it is useful at least for a first analysis to choose a computational method which aims directly at these off-diagonal anharmonicities and thereby allows a correction of the result of

$$
E_{v i b}(\underline{\mathrm{v}})=\Sigma_{\mathrm{i}} \omega_{\mathrm{i}}\left(\mathrm{v}_{\mathrm{i}}+1 / 2\right)-\Sigma_{\mathrm{i}<\mathrm{j}} x_{i j}\left(\mathrm{v}_{\mathrm{i}}+1 / 2\right)\left(\mathrm{v}_{\mathrm{j}}+1 / 2\right)
$$

formula

This formula would break down in the presence of resonant coupling and for very large amplitude motions where the energy levels do not follow a regular scheme. With the sign convention used above the $x_{i j}$ are usually positive for ordinary molecules. For a single quantum excitation of any mode $i$ starting from the ground state $\left(\right.$ all $\mathrm{v}_{\mathrm{j}}=0$ ) and leading to $\mathrm{v}_{\mathrm{i}}=1, \mathrm{v}_{\mathrm{j} \neq \mathrm{i}}=0$, we obtain Eqn. (2) where the second part collects terms which can be obtained from a onedimensional anharmonic treatment without

a one-dimensional anharmonic calculation. We have constructed two-dimensional cuts through the complete potential surfaces by scanning the donor stretch potential $V(r ; q)$ for several values of the relevant intermolecular coordinate $q$. Solution of the one-dimensional Schrödinger equation for the donor stretch by a variational method [36] at each value of $q$ yields vibrational eigenvalues $E^{s}(q), E^{s}{ }_{1}(q), \ldots$ which represent adiabatic surfaces $V^{a d}\left(q ; v_{s}\right)$ for motion along the coordinate $q$ with a given stretch quantum number $\mathrm{v}_{s}$. In a second step we

$$
v_{\mathrm{i}}=\omega_{\mathrm{i}}-2 x_{i i}-1 / 2 \Sigma_{\mathrm{i} \neq \mathrm{j}} x_{i j}=v_{\mathrm{i}}^{\mathrm{anh}, 1 \mathrm{D}}-1 / 2 \Sigma_{\mathrm{i} \neq \mathrm{j}} x_{i j}
$$

coupling into the quantity $v_{i}^{\text {anh, } 1 \mathrm{D}}$

The aforementioned stiffening of the in- solve the Schrödinger equation along the intermolecular coordinate $q$ to obtain eigenvalues $E\left(\mathrm{v}_{q} ; \mathrm{v}_{s}\right)$ within the adiabatic approximation. These sets of eigenvalues can then be analyzed according to Eqn. (1) to provide a set of anharmonic constants. This concept can be extended to the construction of multidimensional adiabatic intermolecular surfaces with parametric dependence on intramolecular excitations as has been done previously for the HF dimmer [6].

\section{Results and Discussion}

\subsection{Potential Surfaces}

In order to illustrate the intrinsic coupling between intramolecular and intermolecular motions in this type of complexes Fig. 2 shows a section of the potential surface of thiirane-HF along the normal coordinates $q_{s}$ and $q_{\sigma}$ which correspond essentially to the $\mathrm{HF}$ and the intermolecular stretch coordinates. Positive values of both normal coordinates correspond to increasing distances and the point $(0,0)$ represents the equilibrium geometry. The tightening of the contour lines towards the top of the graph very nicely shows the approach to the unperturbed higher frequency free HF oscillator. Inspection of the graph also shows that excitation of the $q_{s}$ HF mode, which leads to an increased expectation value $\left\langle q_{s}\right\rangle$ tightens the contour lines for the motion along $q_{\sigma}$ just as increasing the expectation value of $\left\langle q_{\sigma}\right\rangle$ by excitation of the intermolecular stretch brings the $q_{s}$ motion closer to the free HF limit. This behavior is at the origin of the increase of the frequency of any of the involved modes upon excitation of another which causes the blue-shifted hot band sequences accompanying the cold HF excitation [17].

The only exception to this systematic blue shift is observed for the tunneling motion in $\mathrm{HF}-\mathrm{H}_{2} \mathrm{O}$, where the stiffening of the intermolecular interaction upon HF excitation increases the barrier substantially from the dynamically irrelevant electronic barrier height of $157 \mathrm{~cm}^{-1}$ to $186 \mathrm{~cm}^{-1}$ for HF in its ground state and $263 \mathrm{~cm}^{-1}$ in the first excited state. The height of the adiabatic barrier increases roughly in proportion to the HF quantum number. Increasing barriers lead to higher frequencies for ordinary small amplitude motions confined to a single well but they quench tunneling motions in a dramatic way. We consequently predict a ground state tunneling splitting of $49 \mathrm{~cm}^{-1}$ to be compared to only $34 \mathrm{~cm}^{-1}$ for excited HF. Higher excitations of the inversion mode which are well above the adiabatic barrier show the usual frequency increase. This turnaround in the hot band progression leads us to the prediction that one satellite should be observed $15 \mathrm{~cm}^{-1}$ on the red side of the cold HF excitation while all other hot bands fall on the blue side. This prediction 


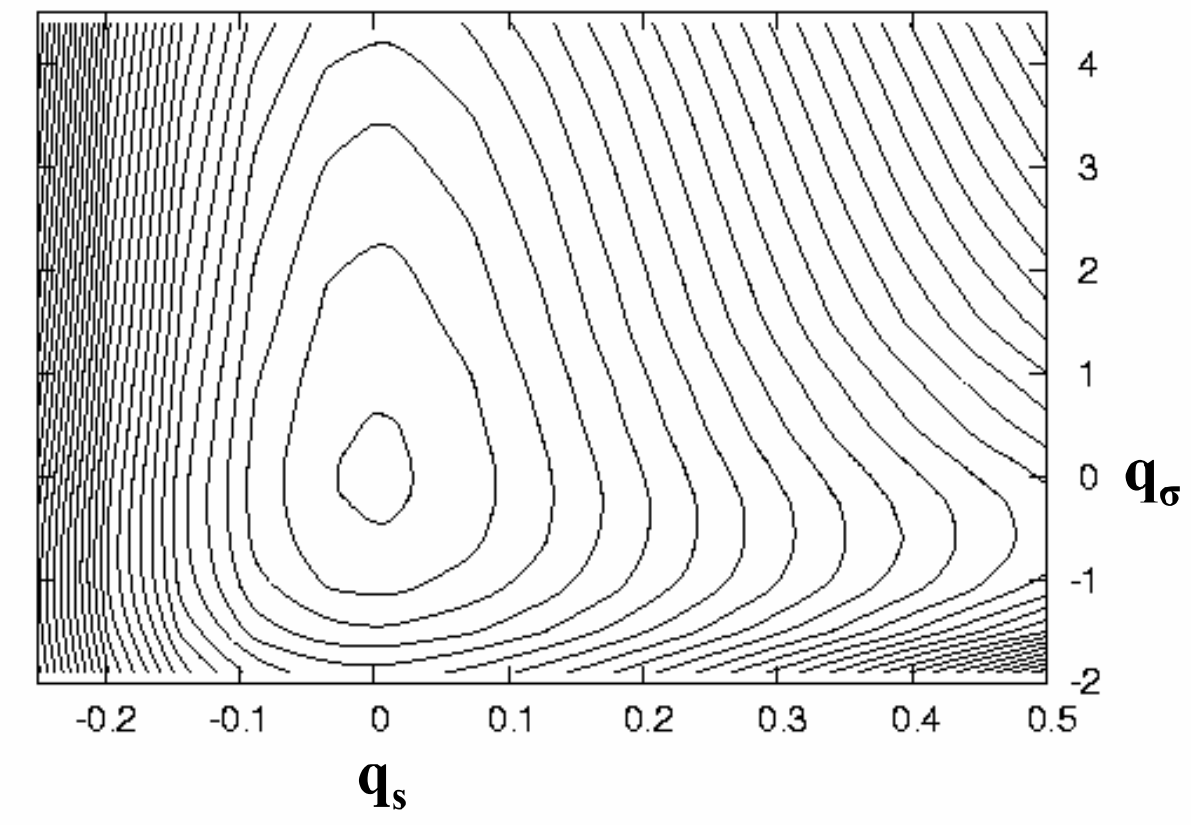

Fig. 2. Cut through the potential surface of thiirane-HF along the normal coordinates corresponding to intramolecular HF stretch $\left(q_{s}\right)$ and intermolecular stretch $\left(q_{\sigma}\right)$.

is consistent with a recent observation of the gas phase spectrum of $\mathrm{HF}-\mathrm{H}_{2} \mathrm{O}$ by Tokhadze and coworkers [37] and preliminary observations in a free jet in our laboratory.

\subsection{Anharmonicities and Vibrational Red-Shifts}

The adiabatic analysis of the dependence of the intermolecular frequencies on excitation of the HF donor stretch yields a set of off-diagonal anharmonicities which is in rather good agreement with the available experimental data considering the limitations imposed by the size of the complex. For thiirane-HF we obtain from the MP2surface $x_{s \sigma}=-28.8 \mathrm{~cm}^{-1}$ (exp. -23.2$)$ and $x_{s \delta 1}=-13.0 \mathrm{~cm}^{-1}$ (exp. -9.5) which clearly supports our experimental analysis [17]. For $\mathrm{HF}-\mathrm{H}_{2} \mathrm{O}$ the analysis of adiabatic vibrational data from the $\operatorname{CCSD}(\mathrm{T})$-surface gives $x_{s \sigma}=-25 \mathrm{~cm}^{-1}, x_{s \delta l}=-13.5 \mathrm{~cm}^{-1}, x_{s l}$ $=-88 \mathrm{~cm}^{-1}$, and $x_{s l 2}=-76 \mathrm{~cm}^{-1}$ but experimental values are unfortunately not yet available. There is, however, a clear correspondence between the two data sets which supports our interpretation of these couplings as the manifestation of a general interaction mechanism.

The combination of the aug-cc-pVTZ basis with the MP2-method gives a value of $3954.6 \mathrm{~cm}^{-1}$ (MP3: 4050.6, MP4: 3921.0) for the free HF stretching frequency in satisfactory agreement with the experimental value of $3961.4 \mathrm{~cm}^{-1}$. Better agreement can be achieved only with much larger basis sets which cannot be used for the thiiraneHF complex. The simple-minded prediction of the vibrational frequency of the HF mode in thiirane-HF using the cut through the MP2-surface along $q$ leads to a value of $3365.4 \mathrm{~cm}^{-1}$ (exp. $3497.5 \mathrm{~cm}^{-1}$ ) which corresponds to a calculated anharmonic red shift of $589.2 \mathrm{~cm}^{-1}$. The experimental value of $463.9 \mathrm{~cm}^{-1}$ is much better reproduced by the harmonic red shift of $482.2 \mathrm{~cm}^{-1}$.

This apparent strong disagreement has essentially two reasons: There are clearly remaining inaccuracies in the $a b$ initio treatment, which we expect to overestimate the intermolecular interaction and in return overestimate the perturbation of the HF oscillator. For instance the chosen combination of basis set and method slightly overestimates the dipole moments of both $\mathrm{HF}$ and thiirane. Our BSSE-corrected dissociation energy of $D_{0}=2001 \mathrm{~cm}^{-1}$ is, however, very close to our recent experimental estimate of $1946 \mathrm{~cm}^{-1}$ [17]. The much more important contribution comes from the off-diagonal anharmonicities with the intermolecular modes which are not included in this kind of calculation and whose sum enters into the correction towards the blue. Unfortunately the experiments do not provide a full set of $x_{i j}$ but the available experimental and theoretical values and the systematically observed relation between $v_{q}$ and $x_{s q}$ allows us to give an estimate of the total effect. For thiirane this contribution turns out to be about $80 \mathrm{~cm}^{-1}$ leading to a red shift with approximate inclusion of coupling effects of $509 \mathrm{~cm}^{-1}$, still not in perfect agreement with experiment, but based on a far more sound accounting for the actual vibrational dynamics than conventional uncoupled treatments and coherent in sign with the remaining electronic structure deficiencies.
The picture is very similar for $\mathrm{HF}-\mathrm{H}_{2} \mathrm{O}$ where the one-dimensional anharmonic prediction based on the $\operatorname{CCSD}(\mathrm{T})$-surface gives $v_{s}=3513 \mathrm{~cm}^{-1}$ (MP2 3467) for the complex. The only published experimental value for the band center is $3608 \mathrm{~cm}^{-1}$ [27] but is probably closer to $3630 \mathrm{~cm}^{-1}$ [37]. Again a major correction arises from the calculated anharmonicities such that the present best estimate for the HF stretching frequency with coupling is $3618 \mathrm{~cm}^{-1}$.

\section{Conclusion}

In spite of the still modest level of theory we find very satisfactory agreement between experimental observations and theoretical models of the coupled vibrations of the hydrogen bond. The similarity of the coupling parameters for several systems strongly suggests a common physical origin for the coupling which therefore has to be included in any treatment of hydrogenbonded systems. More complete dynamical treatments and inclusion of vibrational band strength information will provide a valuable tool to identify the origin of the remaining deficits in the electronic structure technology for the calculation of intermolecular potential energy surfaces of larger complexes. Only by systematically improving the accuracy of both dynamical and electronic structure methods through wellaimed efforts on clearly identified weak points can we hope to arrive at a true understanding of the hydrogen bond in larger systems and its numerous structural implications. The primary goal of theoretical calculations should always be the understanding and not the numerical reproduction of observations.

\section{Acknowledgments}

The authors acknowledge fruitful discussions with P. Asselin and P. Soulard on experimental issues and results and are grateful for an important allocation of supercomputer time at IDRIS through grant no. 031536.

Received: March 15, 2004

[1] G.C. Pimentel, A.C. McClellan, 'The Hydrogen Bond', Freeman, San Francisco, 1960; P. Schuster, G. Zundel, C. Sandorfy, Eds. 'The Hydrogen Bond: Recent Developments in Theory and Experiments', North Holland, Amsterdam, 1976; T.R. Dyke, in 'Hydrogen Bonds', Ed. P. Schuster, Springer, Berlin, 1984; G.A. Jeffrey, W. Saenger, 'Hydrogen Bonding in Biological Structures', Springer, Berlin, 1991.

[2] T.R. Dyke, B.J. Howard, W. Klemperer, J. Chem. Phys. 1972, 56, 2442; M. Quack, M.A. Suhm, Theo. Chim. Acta 1996, 93, 61; J. Bowman, Z. Bačić, Eds. 'Advances in Molecular Vibrations and Collision Dy- 
namic', Vol. III, 'Molecular Clusters', JAI Press, Greenwich, 1998, p. 205.

[3] T.R. Dyke, J.S. Muenter, J. Chem. Phys. 1974, 60, 2929; G.T. Fraser, Int. Rev. Phys. Chem. 1991, 10, 189.

[4] D. Bender, M. Eliades, D.A. Danzeiser, M.W. Jackson, J.W. Bevan, J. Chem. Phys. 1987, 86, 1225; D.D. Nelson, Jr., G.T. Fraser, W. Klemperer, Science 1987, 238, 1670; J.G. Loeser, C.A. Schmuttenmaer, R.C. Cohen, M.J. Elrod, D.W. Steyert, R.J. Saykally, R.E. Bumgarner, G.A. Blake, $J$. Chem. Phys. 1992, 97, 4727; M. Fárník, S. Davis, D.J. Nesbitt, Faraday Discuss. 2001, 118, 63 .

[5] P.R. Bunker, M. Kofranek, H. Lischka, A. Karpfen, J. Chem. Phys. 1988, 89, 3002; M. Quack, M.A. Suhm, Mol. Phys. 1990, 69, 791; C.L. Collins, K. Morihashi, Y. Yamaguchi, H.F. Schaefer III, J. Chem. Phys. 1995, 103, 6051; W. Klopper, M. Quack, M.A. Suhm, J. Chem. Phys. 1998, 108, 10096; D. Feller, J. Chem. Phys. 1992, 96, 6104; C. Millot, A.J. Stone, Mol. Phys. 1992, 77, 439; S. Scheiner, Annu. Rev. Phys. Chem. 1994, 45, 23; R.S. Fellers, L.B. Braly, R.J. Saykally, C. Leforestier, J. Chem. Phys. 1999, 110, 6306.

[6] M. Quack, M.A. Suhm, Chem. Phys. Lett. 1991, 183, 187; M. Quack, M.A. Suhm, J. Chem. Phys. 1991, 95, 28; Q. Wu, D.H. Zhang, J.Z.H. Zhang, J. Chem. Phys. 1995, 103, 2548; D. Luckhaus, M. Quack, U. Schmitt, M.A. Suhm, Ber. Bunsenges. Phys. Chemie 1995, 99, 457; Z. Bačić, Y. Qiu, 'Advances in Molecular Vibrations and Collision Dynamics', Vol. III, 'Molecular Clusters', Eds. J. Bowman, Z. Bačić, JAI Press, Greenwich, 1998, p 183; X.T. Wu, A.B. McCoy, E.F. Hayes, J. Chem. Phys. 1999, 110, 2354; M. Mladenović, M. Lewerenz, Chem. Phys. Lett. 2000, 321, 135; C. Leforestier, F. Gatti, R.S. Fellers, R.J. Saykally, J. Chem. Phys. 2002, 117, 8710.

[7] A.S. Pine, W.J. Lafferty, J. Chem. Phys. 1983, 78, 2154; A.S. Pine, W.J. Lafferty, B.J. Howard, J. Chem. Phys. 1984, 81, 2939; M. Quack, M.A. Suhm, Chem. Phys. Lett. 1990, 171, 517; D.T. Anderson, S. Davis, D.J. Nesbitt, J. Chem. Phys. 1996, 104, 6225; D.T. Anderson, S. Davis, D.J. Nesbitt, J. Chem. Phys. 1996, 105, 4488; S. Davis, D.T. Anderson, J.T. Farrell, Jr., D.J. Nesbitt, J. Chem. Phys. 1996, 104, 8197; K. Nauta, R.E. Miller, J. Chem. Phys. 2000, 113, 10158.

[8] S. Davis, D.T. Anderson, D.J. Nesbitt, $J$. Chem. Phys. 1996, 105, 6645.

[9] A.D. Buckingham, P.W. Fowler, J. Mol. Struct. 1988, 189, 203.

[10] G.E. Ewing, J. Chem. Phys. 1980, 72, 2096.

[11] I.M. Mills, J. Phys. Chem. 1984, 88, 532.

[12] M.D. Marshall, E.J. Bohac, R.E. Miller, J. Chem. Phys. 1992, 97, 3307.

[13] G.M. Chaban, R.B. Gerber, K.C. Janda, J. Phys. Chem. A 2001, 105, 8323. Spectrochim. Acta, Part A 2002, 58, 887.
[14] G.M. Chaban, J.O. Jung, R.B. Gerber, J. Phys. Chem. A 2000, 104, 2772; A. Jansen, M. Gerhards, J. Chem. Phys. 2001, 115, 5445; G.M. Florio, T.S. Zwier, E.M. Myshakin, K.D. Jordan, E.L. Sibert III, $J$. Chem. Phys. 2003, 118, 1735.

[15] A. Milet, T. Korona, R. Moszynski, E. Kochanski, J. Chem. Phys. 1999, 111, 7727.

[16] B. Silvi, R. Wieczorek, Z. Latajka, M.E. Alikhani, A. Dkissi, Y. Bouteiller, J. Chem. Phys. 1999, 111, 6671; M.E. Alikhani, B. Silvi, Phys. Chem. Chem. Phys. 2003, 5, 2494.

[17] M. Goubet, P. Asselin, P. Soulard, M. Lewerenz, Z. Latajka, J. Chem. Phys. 2003, submitted; P. Asselin, M. Goubet, M. Lewerenz, P. Soulard, J.P. Perchard, J. Chem. Phys. 2004, submitted.

[18] R.N. Pribble, A.W. Garrett, K. Haber, T.S. Zwier, J. Chem. Phys. 1995, 103, 531: G. Berden, W.L. Meerts, M. Schmitt, K. Kleinermanns, J. Chem. Phys. 1995, 104, 972; C. Janzen, D. Spangenberg, W. Roth, K. Kleinermanns, J. Chem. Phys. 1999, 110, 9898; A. Inauen, J. Hewel, S. Leutwyler, J. Chem. Phys. 1999, 110, 1463.

[19] J.C. Lassègues, P.V. Huong, Chem. Phys. Lett. 1972, 17, 444; J.C. Lassègues, J. Lascombe, 'Vibrational Spectra and Structure', Vol. 11, Ed. J.R. Durig, Elsevier, Amsterdam, Oxford, New York, 1982.

[20] P. Asselin, B. Dupuis, J.P. Perchard, P. Soulard, Chem. Phys. Lett. 1997, 268, 265; P. Asselin, P. Soulard, M.E. Alikhani, J.P. Perchard, Chem. Phys. 1999, 249, 73; P. Asselin, P. Soulard, M.E. Alikhani, J.P. Perchard, Chem. Phys. 2000, 256, 195.

[21] J.-L. Kuo, M.L. Klein, J. Chem. Phys. 2004, 120, 4690.

[22] C. Lee, C. Sosa, J.J. Novoa, J. Chem. Phys. 1995, 103, 4360; A. Milet, C. Struniewicz, R. Moszynski, P.E.S. Wormer, J. Chem. Phys. 2001, 115, 349; S.M. Hurley, T.E. Dermota, D.P. Hydutsky, A.W. Castleman, Jr., Science 2002, 298, 202; E.M. Cabaleiro-Lago, J.M. Hermida-Ramón, J. Rodríguez-Otero, $J$. Chem. Phys. 2002, 117, 3160; A.J. Huneycutt, R.J. Stickland, F. Hellberg, R.J. Saykally, J. Chem. Phys. 2003, 118, 1221; F. Madeja, M. Havenith, J. Chem. Phys. 2002, 117, 7162

[23] M. Weimann, M. Fárník, M.A. Suhm, Phys. Chem. Chem. Phys. 2002, 4, 3933.

[24] M. M. Szcześniak, S. Scheiner, Y. Bouteiller, J. Chem. Phys. 1984, 81, 5024; Z.-R. Li, D. Wu, Z.-S. Li, X.-R. Huang, F.M. Tao, C.-C. Sun, J. Phys. Chem. 2001, 105, 1163.

[25] L. Andrews, G.L. Johnson, J. Chem. Phys. 1983, 79, 3670.

[26] Z. Kisiel, A.C. Legon, D.J. Millen, Proc. Roy. Soc. London Ser. A 1982, 381, 419; Z. Kisiel, A.C. Legon, D.J. Millen, J. Chem. Phys. 1983, 78, 2910; Z. Kisiel, A.C. Legon, D.J. Millen, J. Mol. Spectrosc.
1984, 112,1

[27] R.K. Thomas, Proc. Roy. Soc. London Ser. A 1975, 344, 579.

[28] Z. Kisiel, B.A. Pietrewicz, P.W. Fowler, A.C. Legon, E. Steiner, J. Phys. Chem. A 2000, 104, 6970.

[29] R. Visvanathan, T.R. Dyke, J. Chem. Phys. 1982, 77, 1166; L.C. Willoughby, A.J. Fillery-Travis, A.C. Legon, J. Chem. Phys. 1984, 81, 20; R.T. Arlinghaus, L. Andrews, Inorg. Chem. 1985, 24, 1523. M.M. Szcześniak, S. Scheiner, J. Chem. Phys. 1985, 83, 1778; M. Tashikawa, K. Iguchi, J. Chem. Phys. 1994, 101, 3062; L. Zhi-Ru, W. Di, L. Ze-Sheng, H. Xu-Ri, F.M. Tao, S. Chia-Chung, J. Phys. Chem. A 2001, 105, 1163 .

[30] M.J. Frisch, G.W. Trucks, H.B. Schlegel, G.E. Scuseria, M.A. Robb, J.R. Cheeseman, V.G. Zakrzewski, J.J.A. Montgomery, R.E. Stratmann, J.C. Burant, S. Dapprich, J.M. Millam, A.D. Daniels, K.N. Kudin, M.C. Strain, O. Farkas, J. Tomasi, V. Barone, M. Cossi, R. Cammi, B. Mennucci, C. Pomelli, C. Adamo, S. Clifford, J. Ochterski, G.A. Petersson, P.Y. Ayala, Q. Cui, K. Morokuma, D.K. Malick, A.D. Rabuck, K. Raghavachari, J.B. Foresman, J. Cioslowski, J.V. Ortiz, A.G. Baboul, B.B. Stefanov, G. Liu, A. Liashenko, P. Piskorz, I. Komaromi, R. Gomperts, R.L. Martin, D.J. Fox, T. Keith, M.A. Al-Laham, C.Y. Peng, A. Nanayakkara, C. Gonzalez, M. Challacombe, P.M.W. Gill, B.G. Johnson, W. Chen, M.W. Wong, J.L. Andres, M. HeadGordon, E.S. Replogle, J.A. Pople, Gaussian 98 (Revision A.8); Gaussian, Inc., Pittsburgh, PA, 1998.

[31] R.A. Kendall, T.H. Dunning, Jr., R.J. Harrison, J. Chem. Phys. 1992, 96, 6796; T.H. Dunning, Jr., K.A. Peterson, 'Encyclopedia of Computational Chemistry', Vol. 1, Ed. P. von Ragué-Schleyer, Wiley, New York, 1998, p. 88.

[32] Molpro, a package of ab initio programs designed by H.-J. Werner and P. J. Knowles, version 2002.1; R.D. Amos, A. Bernhardsson, A. Berning, P. Celani, D.L. Cooper, M.J.O. Deegan, A.J. Dobbyn, F. Eckert, C. Hampel, G. Hetzer, P.J. Knowles, T. Korona, R. Lindh, A.W. Lloyd, S.J. McNicholas, F.R. Manby, W. Meyer, M.E. Mura, A. Nicklass, P. Palmieri, R. Pitzer, G. Rauhut, M. Schütz, U. Schumann, H. Stoll, A.J. Stone, R. Tarroni, T. Thorsteinsson, H.-J. Werner, 2002.

[33] M. Goubet, M. Lewerenz, 2004, to be published.

[34] B. Madebène, M. Lewerenz, 2004, to be published.

[35] M. Mladenović, J. Chem. Phys. 2000, 112, 1070

[36] U. Kleinekathöfer, M. Lewerenz, M. Mladenović, Phys. Rev. Lett. 1999, 83, 4717.

[37] V.P. Bulychev, E.I. Gromova, K.G. Tokhadze, Opt. Spectrosc. 2004, 96, 579. 